\title{
A breathing zirconium metal-organic framework with reversible loss of crystallinity by correlated nanodomain formation**
}

\author{
Bart Bueken, Frederik Vermoortele, Matthew J. Cliffe, Michael T. Wharmby, Damien Foucher, Jelle \\ Wieme, Louis Vanduyfhuys, Charlotte Martineau, Norbert Stock, Francis Taulelle, Veronique Van \\ Speybroeck, Andrew L. Goodwin and Dirk De Vos*
}

\begin{abstract}
The isoreticular analogue of the metal-organic framework UiO-66(Zr), synthesized with the flexible trans-1,4cyclohexanedicarboxylic acid as linker, shows a peculiar breathing behavior by reversibly losing long-range crystalline order upon evacuation. The underlying flexibility is attributed to a concerted conformational contraction of up to two thirds of the linkers, which breaks the local lattice symmetry. X-ray scattering data are well described by a nanodomain model in which differently-oriented tetragonal-type distortions propagate over about 7-10 unit cells.
\end{abstract}

The field of metal-organic frameworks (MOFs) has greatly matured over the past decade, with many developing applications. ${ }^{[1-5]}$ Among MOFs, the zirconium carboxylates stand out for their high thermal and chemical stability, ${ }^{[6]}$ but also because, except for a few cases, ${ }^{[7-10]}$ they all share a similar chemistry based on the $\left[\mathrm{Zr}_{6} \mathrm{O}_{4}(\mathrm{OH})_{4}\left(\mathrm{CO}_{2}\right)_{12}\right]$ inorganic node. ${ }^{[11,12]}$ This allows for a rational synthesis of certain topologies by selecting the appropriate, often rigid aromatic linkers. ${ }^{[13]}$ The resulting materials feature tunable, but static permanent porosity.

[*] B. Bueken, Dr. F. Vermoortele, Prof. Dr. D. De Vos Centre for Surface Chemistry and Catalysis, KULeuven Celestijnenlaan 200F, p.o. box 2461

3001 Leuven, Belgium

E-mail: dirk.devos@biw.kuleuven.be

Dr. M. J. Cliffe, Prof. Dr. A. L. Goodwin

Inorganic Chemistry Laboratory, University of Oxford

South Park Road, Oxford, OX1 3QR, United Kingdom

Dr. M. T. Wharmby, Prof. Dr. N. Stock

Institut für Anorganische Chemie, CAU Kie

Max-Eyth-Straße 2, 24118 Kiel, Germany

Dr. M. T. Wharmby

Diamond Light Source, Harwell Science \& Innovation Campus,

Didcot, OX11 0DE, United Kingdom

D. Foucher, Dr. C. Martineau, Prof. Dr. F. Taulelle

Institut Lavoisier, UMR CNRS 8180

Université de Versailles St-Quentin-en-Yvelines

Avenue des Etats-Unis 45, 78035, Versailles, France

J. Wieme, Dr. L. Vanduyfhuys, Prof. Dr. V. Van Speybroeck

Center for Molecular Modeling, Ghent University

Technologiepark 903, 9052 Zwijnaarde, Belgium

[**] BB, JW, LV, VVS and DDV acknowledge the FWO Flanders, KULeuven (Methusalem grant CASAS and IAP 7/05 Functional Supramolecular Systems), the IWT (SBO-project MOFShape), Ghent University and the ERC (grant 647755) for financial support. MJC and ALG acknowledge the ERC (grant 279705). MTW and NS thank the DFG (SPP 1362) and the EU (FP7/2007-2013, grant 228862) for financial support. Parts of this research were carried out at the light source PETRA III at DESY, a member of the Helmholtz Association (HGF). We thank Dr. U. Ruett and O. Gutowski for assistance in using beamline P07.

Supporting information for this article is given via a link at the end of the document.
One property that remains surprisingly elusive to realize in $\mathrm{Zr}$ MOFs is a dynamic, stimulus-dependent porosity. Such materials, also called breathing or third-generation MOFs, reversibly undergo a phase change in response to pressure, temperature or selective guest adsorption, and are considered ideal candidates for sensing or separations. ${ }^{[14-18]}$

Flexibility in MOFs often originates at the connection between linker and inorganic node. With rigid carboxylates, a typical mechanism is the 'knee-cap', or linker rotation around the axis through the coordinating oxygen atoms. ${ }^{[18]} \mathrm{Zr}$-MOFs however are not predisposed to be flexible in this way due to their often high connectivity and the presence of odd-numbered cycles in many topologies. One way to circumvent these issues is to shift flexibility to the linkers. ${ }^{[14-16]}$ Recently Farha et al. ${ }^{[19]}$ employed this strategy by using large tetrapodal linkers with bendable arms to synthesize breathing Zr-MOFs.

Flexible, aliphatic linkers could both circumvent the topological constraints on breathing related to their rigid counterparts and enable a guest-selective uptake. However, the only aliphatic $\mathrm{Zr}$ MOFs reported to date, based on adipate, irreversibly collapse upon evacuation, ruling out any breathing. ${ }^{[20]}$

Here, we present the synthesis of a flexible $\mathrm{Zr}$-MOF by employing trans-1,4-cyclohexanedicarboxylic acid $\left(\mathrm{H}_{2} \mathrm{Cdc}\right)$ as linker. We successfully synthesized $\mathrm{ZrCDC}$, an aliphatic analogue of the widely studied Zr-terephthalate UiO-66 $\left(\left[\mathrm{Zr}_{6} \mathrm{O}_{4}(\mathrm{OH})_{4}\left(\mathrm{C}_{8} \mathrm{H}_{4} \mathrm{O}_{4}\right)_{6}\right]\right) .{ }^{[12]} \mathrm{ZrCDC}$ displays a unique breathing behavior with a loss of long-range order in the transition from a cubic, adsorbate-filled form to a lower symmetry evacuated phase. As-prepared ZrCDC shows excellent crystallinity (Figure S1-S2), which is retained upon solvent exchange of DMF occupying the pores for ethanol or water. The structure of ZrCDC was solved by Rietveld refinement of a model derived from the UiO-66 framework against a high-resolution $\mathrm{X}$-ray diffraction pattern collected from a water-exchanged sample (Figure S3, Table S3). $\mathrm{ZrCDC}\left(\left[\mathrm{Zr}_{6} \mathrm{O}_{4}(\mathrm{OH})_{4}\left(\mathrm{C}_{8} \mathrm{H}_{10} \mathrm{O}_{4}\right)_{6}\right]\right)$ is an isostructural analogue of UiO-66, and consists of hexanuclear Zr-clusters (Figure 1a) which are bound to twelve neighboring clusters through the ditopic trans-1,4-cyclohexanedicarboxylate ( $\left.\mathrm{cdc}^{2-}\right)$ linkers. The clusters organize in an fcu topology, creating a porous framework with tetrahedral and octahedral cages (Figure 1b-d) of approximately $4.4 \AA$ and $10.1 \AA$ in diameter.

Although ZrCDC only decomposes at temperatures above $275^{\circ} \mathrm{C}$ (Figure S5), guest removal, even by evaporation at room temperature, results in the disappearance of clearly defined Bragg reflections in ZrCDC's X-ray powder diffraction pattern (Figure 2). This loss of long-range crystalline order is also 
a
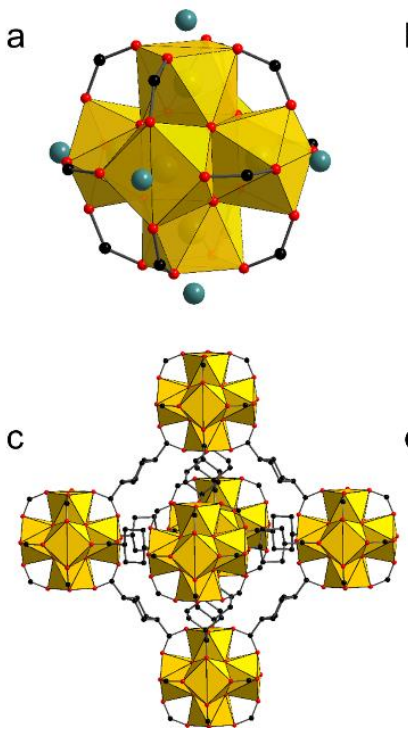

d

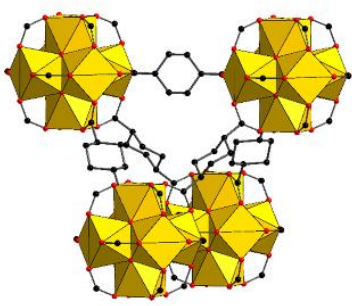

Figure 1. Structure of $\mathrm{ZrCDC}$. $\left[\mathrm{Zr}_{6} \mathrm{O}_{4}(\mathrm{OH})_{4}\left(\mathrm{CO}_{2}\right)_{12}\right]$ clusters (a) arrange in an fcu topology (b), containing octahedral (b, blue; $c)$ and tetrahedral (b, green; $d)$ cages. Water sorption site in (a) indicated in light blue. $\mathrm{O}=\mathrm{red}, \mathrm{C}=$ black, $\mathrm{Zr}=$ yellow. Guests and $\mathrm{H}$-atoms omitted for clarity.

accompanied by a collapse of ZrCDC's pore volume (Figure S6). However, the persistence of broad diffraction features at low angles hints at an enduring short-range order within the material. Moreover, immersion of the evacuated powder in ethanol or water results in the complete recovery of the crystalline lattice. Counterintuitively, apolar solvents such as hexane are unable to induce pore opening, in spite of the hydrophobic nature of the linkers, which points to a guest-specific breathing behavior (Figure S7). Understanding this reversible desorption-induced amorphisation clearly requires insight into the nature of the collapsed phase, hereafter denoted $\mathrm{ZrCDC}_{\mathrm{cp}}$. Therefore, the latter was investigated by a combination of $\mathrm{X}$-ray total scattering, solid state ${ }^{13} \mathrm{C}$ magic angle spinning (MAS) NMR and molecular modeling.

Since the breathing may involve conformational changes of the $\mathrm{cdc}^{2-}$ linker, these were probed by ${ }^{13} \mathrm{C}$ MAS NMR (Figure 3 ). In accordance with their Rietveld refined structure, water- and ethanol-exchanged $\mathrm{ZrCDC}$ only contain $\mathrm{cdc}^{2-}$ in its elongated e,econformation, denoted e, (182.0, 45.8 and $29.0 \mathrm{ppm}$ ), with the
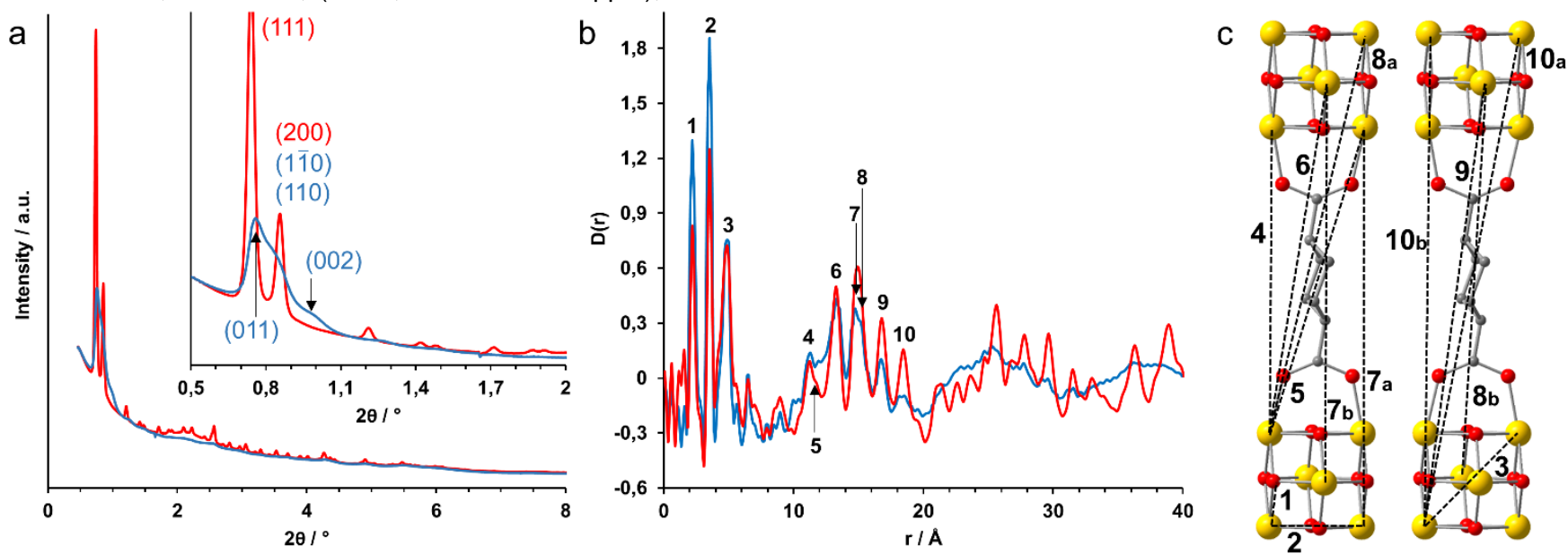

Figure 2. (a) Powder X-ray diffraction data $\left(\lambda=0.1551 \AA\right.$ ) and (b) pair distribution function $\mathrm{D}(\mathrm{r})$ of ethanol-loaded $\mathrm{ZrCDC}$ (red trace) and $\mathrm{ZrCDC} \mathrm{C}_{\mathrm{cp}}(\mathrm{blue}$ trace) Characteristic reflections for each phase are indicated. (c) Characteristic Zr-Zr distances indicated in the PDF. $\mathrm{O}=\mathrm{red}, \mathrm{C}=\mathrm{gray}, \mathrm{Zr}=\mathrm{yellow}$. 


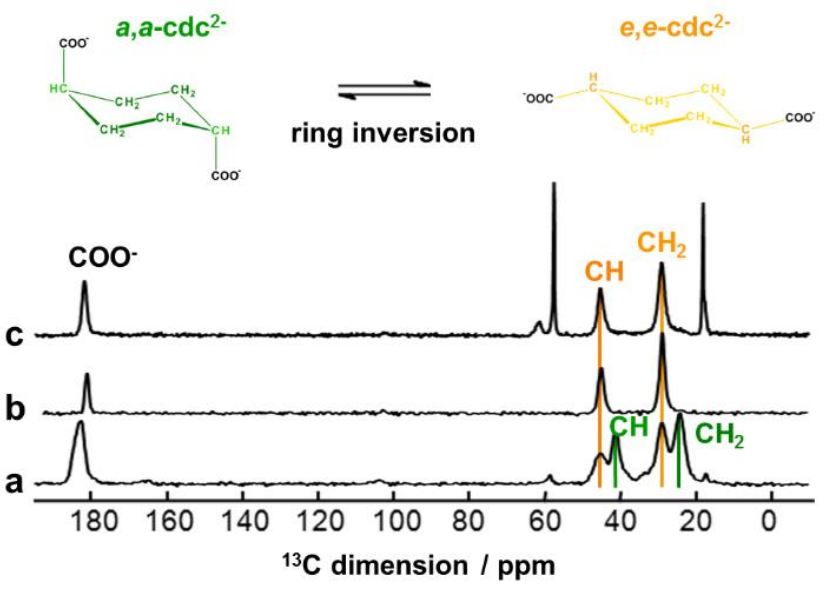

Figure 3. ${ }^{13} \mathrm{C}$ MAS NMR spectra of $\operatorname{ZrCDC}_{\mathrm{cp}}$ (a), and $\mathrm{ZrCDC}$ after water (b) and ethanol adsorption (c). Besides $e, e-\mathrm{cdc}^{2-}\left(\mathrm{CH}_{2}=29.0 \mathrm{ppm}, \mathrm{CH}=45.8 \mathrm{ppm}\right)$, $\mathrm{ZrCDC}_{\mathrm{cp}}$ also contains a, $a-\mathrm{cdc}^{2-}\left(\mathrm{CH}_{2}=24.3 \mathrm{ppm}, \mathrm{CH}=41.5 \mathrm{ppm}\right)$ in a maximal ratio of $2: 1$ to e,e-cdc ${ }^{2-}$. Ethanol $\mathrm{CH}_{3}=18.1 \mathrm{ppm}, \mathrm{CH}_{2}=57.8 \mathrm{ppm}$.

unit cell to a lower symmetry cell upon drying. A satisfactory Pawley fit of $\mathrm{ZrCDC}_{\mathrm{cp}}$ 's diffraction pattern was obtained in the tetragonal $14 / \mathrm{mmm}$ space group, which is the highest-symmetry subgroup of the parent $F m \overline{3} m$ space group for which we could obtain a good fit to data (Figure 4c). Following contraction to a body-centered tetragonal cell (Figure S10), the cubic (111) reflection $(\mathrm{d}=12.0 \AA)$ is conserved as the $(011)$ reflection $(\mathrm{d}=$ $11.7 \AA)$. The $(200)$ reflection $(d=10.4 \AA)$ however has split intothe tetragonal $(1 \overline{1} 0)$ and $(110)(d=10.6 \AA)$ and $(002)(d=8.9 \AA)$ reflections. A similar splitting of the (200) reflection has previously been reported for a tetragonal variant of UiO-66- $\mathrm{NH}_{2} \cdot{ }^{[24]}$ Furthermore, the intensity ratios in the diffraction pattern suggest that the distances between the cubic $\{001\}$ planes have shortened by approximately $3 \AA$ in $\mathrm{ZrCDC}_{\mathrm{cp}}$. Geometrically, this would correspond to a contraction of two thirds of the linkers from $e$ to a along the [001] direction, in accordance with the observed a:e ratio in NMR. Combining all our observations, we geometrically constructed a tetragonally-distorted model for $\mathrm{ZrCDC}_{\mathrm{cp}}$ (Figure $4 \mathrm{a}$, Figures S11-S13), which maintains the topology and connectivity of $\mathrm{ZrCDC}$ : each cluster now connects to twelve neighbors via four e-linkers in the (002) plane and eight contracted a-linkers above and below this plane.

To correctly fit the broad reflections in $\mathrm{ZrCDC}_{\mathrm{cp}}$ 's powder pattern, a Lorentzian size broadening term was introduced, from which a crystalline domain size of approximately $13.4(1.3) \mathrm{nm}$ or $7-10$ tetragonal unit cells was deduced. This domain formation originates from the fact that ZrCDC's cubic unit cell can contract along the three equivalent $\langle 100\rangle$ directions to end up in the correct tetragonal cell. While the direction along which one cell contracts is correlated to the direction selected by its neighbors the contraction does not propagate through the whole crystal in the same direction. Rather, randomly oriented correlated nanodomains are formed in which short-range order is preserved, with a concomitant loss of the long-range crystalline order of ZrCDC. This behavior was simulated as a cluster growth model (Figure $4 b){ }^{[25]}$ which yields a calculated diffraction pattern that reproduces the key features of $\mathrm{ZrCDC}_{\mathrm{cp}}$ 's experimental pattern (Figure 4c). The tetragonal model represents the limiting case for a 2:1 a:e ratio. Lower ratios might be accommodated by alternative types of symmetry lowering (e.g. a rhombohedral-type distortion would correspond to a 1:1 a:e ratio; see Figure S14). While the tetragonal case clearly dominates based on the form of the diffraction profile and the observed linker ratio, it is possible that both cases could be present in $\mathrm{ZrCDC}_{\mathrm{cp}}$, for instance at domain boundaries, resulting in intermediate linker ratios.

To obtain more insight in the relative stability of ZrCDC and $\mathrm{ZrCDC}_{\mathrm{cp}}$, periodic DFT calculations were performed. $\mathrm{ZrCDC}_{\mathrm{cp}}$ is $102 \mathrm{~kJ} \cdot \mathrm{mol}^{-1}$ per cluster more stable than a hypothetical, openpore $\mathrm{ZrCDC}$ framework due to a much larger contribution of stabilizing dispersion interactions in $\mathrm{ZrCDC}_{\mathrm{cp}}$ (Figure S15). To obtain some insight in the dynamic behavior of $\mathrm{ZrCDC}$ and the effect of water adsorption, energies were calculated for the openpore $\mathrm{ZrCDC}$ with a water molecule adsorbed on top of each $\mathrm{Zr}^{4+}$ atom, as observed in the Rietveld refinement (Figure 1a). Each water molecule realizes an energy gain of $38 \mathrm{~kJ} \cdot \mathrm{mol}^{-1}$ by hydrogen bonding to the carboxylates, which indicates that sufficient loading with water could stabilize the open-pore ZrCDC. The hydrophilic nature of the cluster thus likely dominates the breathing behavior, explaining $\mathrm{ZrCDC}_{\mathrm{cp}}$ 's preferential adsorption of hydrogen bond donating molecules like water or ethanol. Interactions between guests and the cluster $\mu_{3}-\mathrm{OH}$ groups could also contribute to this selective uptake.
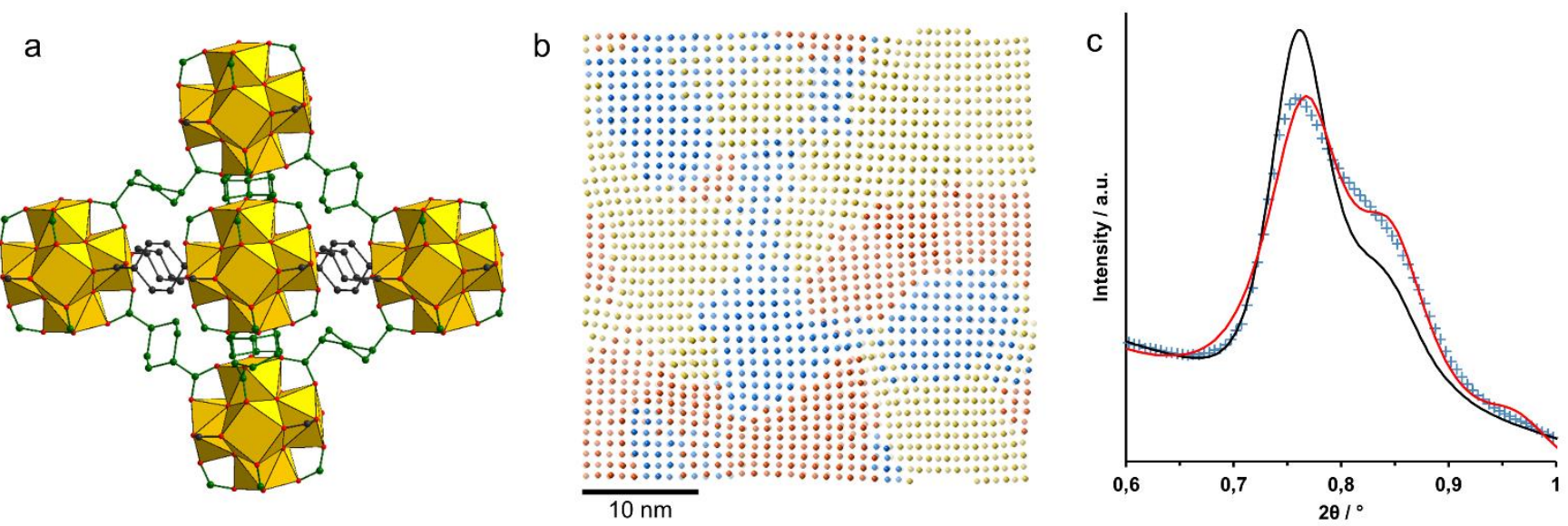

Figure 4. Model of $Z \mathrm{rCDC}_{\mathrm{cp}}$. (a) Contraction of two thirds of the linkers (green) results in a tetragonally-distorted cell. $\mathrm{O}=\mathrm{red}, \mathrm{C}=\mathrm{black} / \mathrm{green}, \mathrm{Zr}=\mathrm{yellow}$. (b) Cluster growth model simulation. Contraction along the three cubic $<100>$ directions yields randomly oriented, short-range ordered tetragonal nanodomains. Dots represent inorganic nodes, colors indicate domains of different orientations. (c) Diffraction pattern for this nodal model (black trace), experimental diffraction pattern of $\mathrm{ZrCDC}_{\mathrm{cp}}$ (blue crosses) and Pawley fit in the tetragonal space group $14 / \mathrm{mmm}$ (red trace; $\mathrm{R}_{\mathrm{wp}}=1.922 \%$ ). 
In summary, we synthesized a breathing analogue of the topologically rigid UiO-66 by using cdc $^{2-}$ as flexible linker. Guest removal results in a tetragonal-type contraction of the crystalline cubic ZrCDC structure, which is accompanied by a loss of longrange order due to the clustering of these tetragonal cells in differently-oriented nanodomains. ZrCDC's breathing is driven by a selective uptake of hydrogen bond donating molecules, which opens opportunities for its use in separations or sensing applications.

Keywords: zirconium $•$ metal-organic frameworks $\bullet$ structure elucidation $\bullet$ phase transitions $\cdot$ dynamic porosity

[1] J.-R. Li, R. J. Kuppler, H.-C. Zhou, Chem. Soc. Rev. 2009, 38, 1477.

[2] L. E. Kreno, K. Leong, O. K. Farha, M. Allendorf, R. P. Van Duyne, J. T. Hupp, Chem. Rev. 2012, 112, 1105.

[3] H. Furukawa, K. E. Cordova, M. O’Keeffe, O. M. Yaghi, Science 2013, 341 1230444.

[4] J. Liu, L. Chen, H. Cui, J. Zhang, L. Zhang, C.-Y. Su, Chem. Soc. Rev. 2014 $43,6011$.

[5] B. Van De Voorde, B. Bueken, J. Denayer, D. De Vos, Chem. Soc. Rev 2014, 43, 5766.

[6] T. Devic, C. Serre, Chem. Soc. Rev. 2014, 43, 6097.

[7] V. Guillerm, F. Ragon, M. Dan-Hardi, T. Devic, M. Vishnuvarthan, B. Campo, A. Vimont, G. Clet, Q. Yang, G. Maurin, et al., Angew. Chem. Int. Ed. 2012, $51,9267$.

[8] D. Feng, H.-L. Jiang, Y.-P. Chen, Z.-Y. Gu, Z. Wei, H.-C. Zhou, Inorg. Chem 2013, 52, 12661.

[9] L. Cooper, N. Guillou, C. Martineau, E. Elkaim, F. Taulelle, C. Serre, T. Devic, Eur. J. Inorg. Chem. 2014, 2014, 6281.

[10] G. Mouchaham, L. Cooper, N. Guillou, C. Martineau, E. Elkaïm, S. Bourrelly, P. L. Llewellyn, C. Allain, G. Clavier, C. Serre, et al., Angew. Chem. 2015 127,13495

[11] G. Kickelbick, U. Schubert, Chem. Ber. 1997, 130, 473.

[12] J. H. Cavka, S. Jakobsen, U. Olsbye, N. Guillou, C. Lamberti, S. Bordiga K. P. Lillerud, J. Am. Chem. Soc. 2008, 130, 13850.

[13] H. Furukawa, F. Gándara, Y.-B. Zhang, J. Jiang, W. L. Queen, M. R. Hudson, O. M. Yaghi, J. Am. Chem. Soc. 2014, 136, 4369.

[14] S. Kitagawa, R. Kitaura, S. Noro, Angew. Chem. Int. Ed. Engl. 2004, 43 2334

[15] Z.-J. Lin, J. Lü, M. Hong, R. Cao, Chem. Soc. Rev. 2014, 5867.

[16] A. Schneemann, V. Bon, I. Schwedler, I. Senkovska, S. Kaskel, R. A Fischer, Chem. Soc. Rev. 2014, 6062

[17] S. Horike, S. Shimomura, S. Kitagawa, Nat. Chem. 2009, 1, 695.

[18] G. Férey, C. Serre, Chem. Soc. Rev. 2009, 38, 1380.

[19] P. Deria, D. A. Gomez-Gualdron, W. Bury, H. T. Schaef, T. C. Wang, P. K. Thallapally, A. A. Sarjeant, R. Q. Snurr, J. T. Hupp, O. K. Farha, J. Am. Chem. Soc. 2015, 13183

[20] H. Reinsch, I. Stassen, B. Bueken, A. Lieb, R. Ameloot, D. De Vos, CrystEngComm 2014, 17, 331.

[21] F. Niekiel, M. Ackermann, P. Guerrier, A. Rothkirch, N. Stock, Inorg. Chem. 2013, 52, 8699.

[22] B. Bueken, F. Vermoortele, D. E. P. Vanpoucke, H. Reinsch, C.-C. Tsou, P. Valvekens, T. De Baerdemaeker, R. Ameloot, C. E. A. Kirschhock, V. Van Speybroeck, et al., Angew. Chem. 2015, 14118.

[23] F. Niekiel, J. Lannoeye, H. Reinsch, A. S. Munn, A. Heerwig, I. Zizak, S. Kaskel, R. I. Walton, D. De Vos, P. Llewellyn, et al., Inorg. Chem. 2014, 53 4610.

[24] H. Reinsch, B. Bueken, F. Vermoortele, I. Stassen, A. Lieb, K.-P. Lillerud, D. De Vos, CrystEngComm 2015, 17, 4070.

[25] M. J. Cliffe, W. Wan, X. Zou, P. A. Chater, A. K. Kleppe, M. G. Tucker, H. Wilhelm, N. P. Funnell, F.-X. Coudert, A. L. Goodwin, Nat. Commun. 2014 5,4176 


\section{COMMUNICATION}

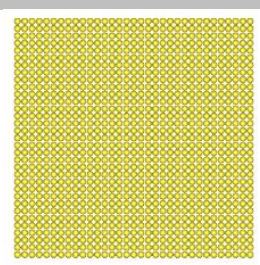

long-range order

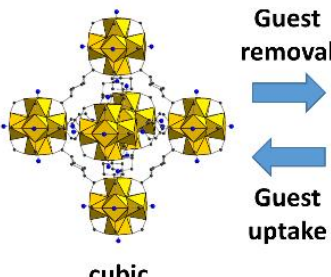

cubic

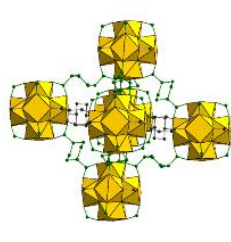

tetragonal

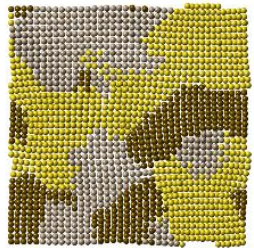

short-range order

Camouflaged from diffraction: The aliphatic isoreticular analogue of the well-known MOF UiO-66(Zr) undergoes a unique and reversible phase change upon guest removal, which involves a topology-dictated loss of long-range order through the correlated formation of short-range ordered nanodomains.

Bart Bueken, Dr. Frederik Vermoortele, Dr. Matthew J. Cliffe, Dr. Michael T. Wharmby, Damien Foucher, Jelle Wieme, Dr. Louis Vanduyfhuys, Dr. Charlotte Martineau, Prof. Dr. Norbert Stock, Prof. Dr. Francis Taulelle, Prof. Dr. Veronique Van Speybroeck, Prof. Dr. Andrew L. Goodwin and Prof. Dr. Dirk De Vos

Page No. - Page No.

A breathing zirconium metalorganic framework with reversible loss of crystallinity by correlated nanodomain formation

As published in Chemistry - A European Journal, vol. 22 (10), p. 3264-3267, doi:

10.1002/chem.201600330.

(C) 2016, Wiley-VCH Verlag GmbH \& Co. KGaA, Weinheim 